\title{
Fabrication of a MEMS Micromirror Based on Bulk Silicon Micromachining Combined With Grayscale Lithography
}

\author{
Inês S. Garcia ${ }^{\circledR}$, Carlos Ferreira ${ }^{\circledR}$, Member, IEEE, Joana D. Santos, Marco Martins ${ }^{\circledR}$, Rosana A. Dias, \\ Diogo E. Aguiam ${ }^{\circledR}$, Member, IEEE, Jorge Cabral, Member, IEEE, and João Gaspar, Member, IEEE
}

\begin{abstract}
A 1D MEMS (Micro-Electro-Mechanical Systems) mirror for LiDAR applications, based on vertically asymmetric comb-drive electrostatic actuators, is presented in this work employing a novel fabrication process. This novel micromachining process combines typical SOI-based bulk micromachining and grayscale lithography, enabling the fabrication of combs actuators with asymmetric heights using a single lithography step in the active layer. With this technique, the fabrication process is simplified, and the overall costs are reduced since the number of required lithography steps decrease. The fabricated mirrors present self-aligned electrodes with a $2.8 \mu \mathrm{m}$ gap and asymmetric heights of the movable and the fixed electrodes of $20 \mu \mathrm{m}$ and $50 \mu \mathrm{m}$, respectively. These asymmetric actuators are an essential feature for the operation mode of this device, enabling both in resonant and static mode operation. A mirror field of view (FOV) of $54^{\circ}$ at $838 \mathrm{~Hz}$ was achieved under lowpressure, when resonantly operated, and a FOV of $0.8^{\circ}$ in the static mode.
\end{abstract}

Index Terms-Bulk-micromachining process, grayscale, MEMS mirror, scanner, vertically asymmetric electrodes.

\section{INTRODUCTION}

$\mathbf{N}$ OWADAYS , MEMS mirrors are present in many applications such as endoscopic imaging, fiberoptic communications, micro spectrometers, and laser scanner systems (i.e., LiDAR - Light Detection and Ranging, image projection, displays, and head-up displays [1]). Devices fabricated by surface and bulk micromachining are widely presented in

Manuscript received May 15, 2020; revised June 25, 2020; accepted June 26, 2020. Date of publication July 10, 2020; date of current version October 7, 2020. This work was supported by the European Structural and Investment Funds in the FEDER Component through the Operational Competitiveness and Internationalization Programme (COMPETE 2020) under Project 037902 (POCI-01-0247-FEDER-037902). The work of Carlos Ferreira was supported by the Fundação para a Ciência e Tecnologia (FCT) under Grant PD/BDE/135102/2017. Subject Editor M. Rais-Zadeh. (Corresponding author: Inês S. Garcia.)

Inês S. Garcia, Carlos Ferreira, Rosana A. Dias, Diogo E. Aguiam, and João Gaspar are with the International Iberian Nanotechnology Laboratory (INL), 4715-330 Braga, Portugal, and also with the ALGORITMI Center, University of Minho at Campus Azurém, 4800-058 Guimarães, Portugal (e-mail: ines.garcia@inl.int; carlos.ferreira@inl.int; rosana.dias@inl.int; diogo.aguiam@inl.int; joao.gaspar@inl.int).

Joana D. Santos and Marco Martins are with the International Iberian Nanotechnology Laboratory (INL), 4715-330 Braga, Portugal (e-mail: joana.santos@inl.int; marco.martins@inl.int).

Jorge Cabral is with the ALGORITMI Center, University of Minho at Campus Azurém, 4800-058 Guimarães, Portugal (e-mail: jcabral@dei.uminho.pt).

Color versions of one or more of the figures in this article are available online at http://ieeexplore.ieee.org.

Digital Object Identifier 10.1109/JMEMS.2020.3006746 the literature [2], mainly using electrostatic, electromagnetic, thermal or piezoelectric actuation. Electrostatically actuated MEMS mirrors are typically fabricated in simple fabrication processes, being extremely compact and compatible with other micromachining technologies [1], with the advantage of not requiring rare materials, as is the case of piezoelectric and electromagnetic devices, and have the capability of being actuated at higher frequencies compared to thermal actuation.

MEMS mirrors using electrostatic actuators have been presented in literature, where the primary schemes can be divided into vertical comb actuators and parallel-plate actuators. In general, comb actuation allows for larger mirror angles $(\theta)$ and, consequently, a larger field of view (FOV) when compared to parallel-plate actuation [2], because the electrodes relative positions do not restrain displacement. Vertical combdrive actuators offer key advantages: it does not restrict the maximum deflection angle such as the use of parallel-plates and the pull-in associated with parallel-plates can be avoided. Several ways of combining vertical comb-drive actuators with micromirrors have been presented in the literature, mainly in staggered vertical combs, SVC (same electrode dimensions but with z-axis offset) [3] and [4], angular vertical combs, AVC (same electrode dimensions but with an angular offset) or vertically asymmetric combs-drives (different heights and with z-axis offset) [5] and [6].

Typical vertically asymmetric comb electrodes fabrication relies on the use of several lithography steps to define the masks to fabricate electrodes with thickness asymmetry on a single silicon layer, as presented in [5] that uses different materials to create a two level mask and in [6] that uses two layers of the same material defined in different lithographic processes. These are often prone to misalignments between actuators, and when designing a device with a minimal electrodes gap (smaller than $3 \mu \mathrm{m}$ given a $50 \mu \mathrm{m}$ SOI layer), this misalignment can compromise the final device performance or process yield. More recently, new attempts to develop selfaligned comb electrodes have been presented. In [7], higher alignment tolerance is achieved by using dummy electrodes and SOI-SOI wafer bonding. Other approaches use electrodes with the same thickness and the offset is induced by out-ofplane actuation [3] and [4] or by using suspended actuators and mechanically deflecting those at the expense of complex assembly procedures [8]. 


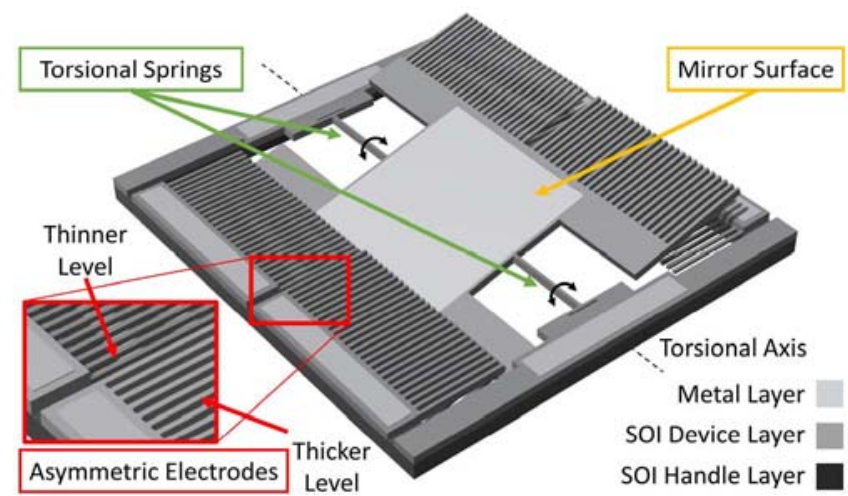

Fig. 1. 3D schematic of the 1D MEMS mirror.

In this work, a 1D (one torsional axis) MEMS mirror is presented employing vertical asymmetric comb-drive electrostatic actuation for automotive LiDAR applications, micromachined using a novel self-aligned, dicing-free [9] and low-cost fabrication process, capable of achieving a $\mathrm{FOV}$ of $54^{\circ}$ under low pressure. This novel fabrication process combines typical SOI-based bulk micromachining and grayscale (GS) lithography to create a single mask with multilevel topography, followed by a sequence of etching and mask thinning steps, creating vertically asymmetric structures enabling a small and self-aligned electrode gap of approximately $2.8 \mu \mathrm{m}$. This process when compared to previous references [3]-[8], presents a simpler and cost-effective process to achieve non-resonant torsional motion using a single layer device.

\section{1D MEMS MirRor MOdEL AND DESIGN}

The MEMS mirror schematic is depicted in Fig. 1 . A $1 \times 1$ $\mathrm{mm}^{2}$ mirror is attached to two torsional springs and four pairs of vertically asymmetric electrodes. The mechanical model that represents the mirror motions is presented in equation (1):

$$
J \ddot{\theta}+B \dot{\theta}+k \theta=T
$$

where $J$ is the mirror moment of inertia, $B$ the damping coefficient, $k$ the torsional spring constant, $T$ the torque necessary to the mirror movement, and $\theta$ the mirror angle. The correspondent natural frequency $f_{0}$ is estimated by the equation (2), and as can be seen, is influenced by the torsional spring elastic constant and the mirror moment of inertia.

$$
f_{0}=\frac{1}{2 \pi} \sqrt{k / J}
$$

Each electrode pair consists of fixed and movable combs actuators, and an electrostatic torque is generated when a voltage is applied between the comb electrodes thus rotating the mirror around the springs' axis. The implementation of vertical comb-drive electrostatic actuators is an essential feature for the operation of this device, enabling it to be operated both in resonant and static mode, unlike the symmetrical actuators which only allow the resonant mode. In resonant operation asymmetric actuators require higher voltages than equivalent symmetric actuators to achieve the same FOV (to achieve the same electrostatic torque given the smaller
TABLE I

\begin{tabular}{|c|c|c|}
\hline \multicolumn{2}{|l|}{ Mechanical Features } & 1D MEMS Mirror \\
\hline Mirror area $(1 \times w)$ & & $1000 \mu \mathrm{m} \times 1000 \mu \mathrm{m}$ \\
\hline Springs dimensions $(1 \times \mathrm{w} \times \mathrm{h})$ & & $600 \mu \mathrm{m} \times 20 \mu \mathrm{m} \times 50 \mu \mathrm{m}$ \\
\hline Number of electrostatic electrodes & & 100 \\
\hline Electrodes dimensions $(1 \times \mathrm{w} \times \mathrm{h})$ & Fixed & $500 \mu \mathrm{m} \times 20 \mu \mathrm{m} \times 50 \mu \mathrm{m}$ \\
\hline & Movable & $500 \mu \mathrm{m} \times 20 \mu \mathrm{m} \times 20 \mu \mathrm{m}$ \\
\hline Electrodes gaps & & $2 \mu \mathrm{m}$ \\
\hline
\end{tabular}

MEMS MirRor DESIGN FEATURES

overlapping area). The device static mode operation is due to the height asymmetry between the movable and fixed in the rest position that enables a vertical displacement of the mirror structure, allowing an out-of-plane, pull in safe motion. When a DC voltage is applied between both electrodes, a constant electrostatic torque is generated, twisting the mirror to a fixed angle around the springs' axis. The higher the DC actuation voltage, the greater the torque applied; until the equilibrium between the torque produced and spring restoring elastic torque. The maximum angle attainable in static mode is geometrically defined. Table. I presents the designed dimensions and mechanical features.

\section{Microfabrication Process}

\section{A. Grayscale Lithography}

Standard lithography is a process that creates a pattern on photo-resist, a photo-sensitive polymeric material, to be used as a sacrificial layer in etching or deposition processes of surface and bulk micromachining. This photoresist is polymerized using ultra-violet light and this exposure can be done either by using a pre-existent hard mask and a collimated light source or using a direct-write-laser (DWL). A development step follows, in which the pattern is revealed by removal of the exposed region (a positive photoresist is assumed, while the unexposed material is removed in the case of a negative photoresist) thus creating a binary level mask and uncovering selected regions of the underlying substrate for further processing.

On the other hand, grayscale (GS) lithography enables the creation of masks with multiple levels of photoresist thickness. In GS lithography, the photoresist layer is exposed using a lateral variation of the exposure dose using an intensity-controlled laser beam. The local development rate of the exposed photoresist changes according to the exposure dose, as represented in Fig. 2, and the desired resist topography is created during the development process, resulting in a multilevel mask with different thickness levels on the photoresist.

Grayscale lithography is often used to pattern micro-lenses, diffractive optical elements, computer-generated holograms, among others [10]. However, this can also be used to create discrete photoresist levels, as shown in Fig. 2 (a), that when combined with a sequential bulk micromachining process, transpose this discrete mask to the substrate. This technique enables the patterning of perfectly aligned (no alignment between separate lithography steps is required) asymmetric 


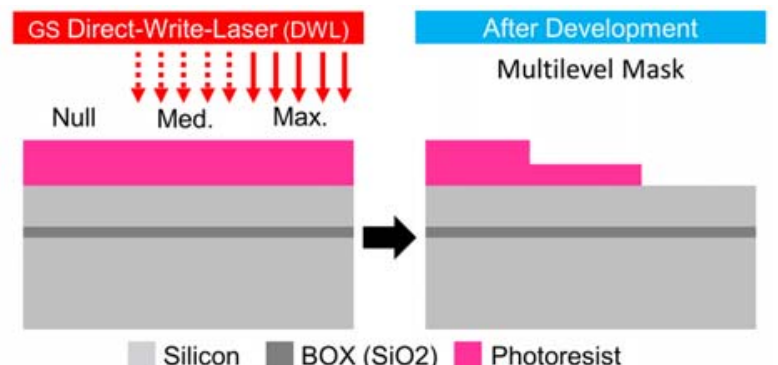

(a)

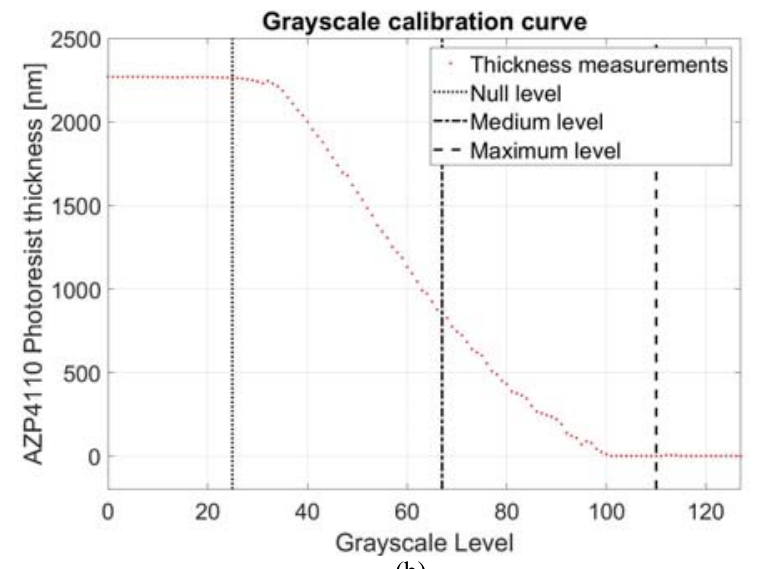

(b)

Fig. 2. GS lithography process schematic (a). Grayscale characterization curve, showing that at least 80 different GS levels are possible (b).

structures that, in this particular application, enables the creation of self-aligned electrostatic combs where each GS level corresponds to a different thickness to be patterned on the silicon substrate. The fact that no alignment between separate lithography steps is required, bypasses most misalignment sources from the lithography process, that may arise due to the resolution of the system camera or from the alignment marks quality, among others.

The final thickness of photoresist after being developed, for each intensity level, was characterized to ensure the optimum laser intensity that would be needed during the process. For this calibration, an initial AZP4110 photoresist layer with a thickness of $2.2 \mu \mathrm{m}$ was used. The calibration curve is shown in Fig. 2 b) and presents over 80 grayscale levels in the linear region defined between thickness $2.2 \mu \mathrm{m}$ and $0 \mu \mathrm{m}$. However, in this particular design, only three GS levels were used to define the asymmetric comb electrodes, which presented a final thickness of $0 \mu \mathrm{m}$ (maximum level), $1.1 \mu \mathrm{m}$ (medium level) and $2.2 \mu \mathrm{m}$ (null level).

\section{B. Micromachining Process}

The devices were fabricated on a $50 \mu \mathrm{m}$-thick SOI wafer, Fig. 3 (a), where initially a thin layer $(500 \mathrm{~nm})$ of $\mathrm{AlSiCu}$ was sputtered and patterned through Reactive Ion Etching (RIE) on the wafer front-side (FS), Fig. 3 (b), to create the device electrical contacts, the mirror coating, and the FS alignment marks. The AlSiCu coating patterned on the mirror surface aims to increase the mirror reflectivity for the near-infrared laser, wavelength used in the LiDAR application.

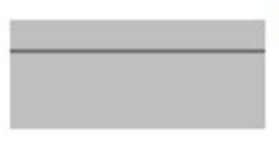

(a)

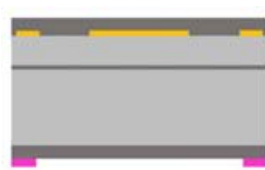

(d)

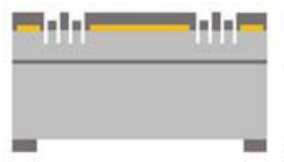

(g)

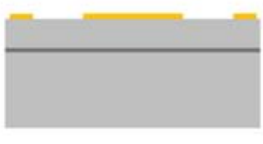

(b)

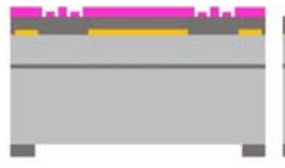

(e)

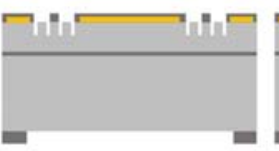

(h)

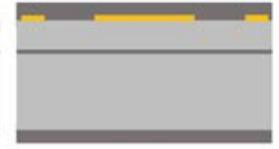

(c)

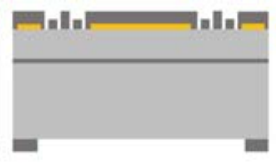

(f)

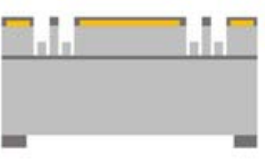

(i)

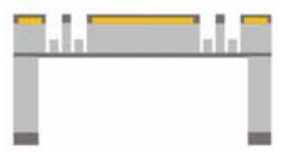

(j)

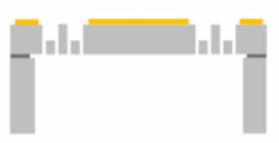

(k)
Silicon $\square$ Silicon Oxide $\square$ Metal $\square$ Resist

Fig. 3. MEMS mirrors main fabrication steps. (a) SOI Wafer; (b) $\mathrm{AlSiCu}$ thin-film patterning; (c) $\mathrm{FS}$ and $\mathrm{BS} \mathrm{SiO}_{2}$ layer deposition; (d) $\mathrm{BS}$ hard-mask lithography; (e) BS hard-mask patterning and FS GS lithography; (f) FS GS hard-mask patterning; (g) FS 1st DRIE; (h) FS GS hard-mask thinning; (i) FS 2nd DRIE; (j) BS DRIE; (k) HF structures release.

With the target interelectrode gap aspect ratio of the MEMS mirror of 25 (on FS, $50 \mu \mathrm{m}$ deep $/ 2 \mu \mathrm{m}$ wide), the difficulty to obtain the multilevel topography in the silicon without removing and damaging the GS photoresist mask increases, given the etch rate of both materials and the resulting selectivity during the etching process. In order to address this constraint and work with a better selectivity rates, hard-mask layers were employed. Thus, a thick layer of silicon oxide $\left(3 \mu \mathrm{m}\right.$ of $\left.\mathrm{SiO}_{2}\right)$ was deposited on both sides of the SOI wafer by PlasmaEnhanced Chemical Vapor Deposition (PECVD), Fig. 3 (c).

The back-side (BS) oxide layer was then patterned by RIE, Fig. 3 (d), to define the BS hard-mask for the subsequent silicon patterning. In this layer, the BS mirror cavities combined with the BS alignment marks (aligned to the front side marks) were patterned. These marks are essential to ensure that the BS mask is aligned with the FS mask already patterned and to ensure a final functional device.

For the FS mirror design (mirror structure, springs, and electrodes) a GS mask with three different thickness of photoresist was exposed, Fig. 3 (e).

The multilevel photoresist mask previously developed was then transferred to the $\mathrm{SiO}_{2}$ layer through a sequential process of silicon oxide etching by RIE and photoresist mask thinning through $\mathrm{O}_{2}$ plasma strip to achieve the multilevel topography on the hard-mask layer, Fig. 3 (f), with three different levels/thicknesses: $3 \mu \mathrm{m}, 1.5 \mu \mathrm{m}$, and $0 \mu \mathrm{m}$. Each mask step height must include extra thickness to compensate for the mask material removed during the etching of the substrate material.

Taking advantage of a high substrate to mask selectivity of the etching process, a sequential iterative etching process 


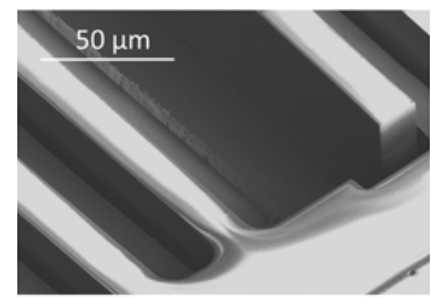

(a)

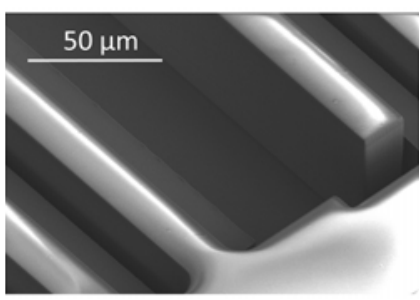

(b)
Fig. 4. SEM images of the thinned electrodes (a) before and (b) after the cleaning of the organic residues due to the DRIE process.

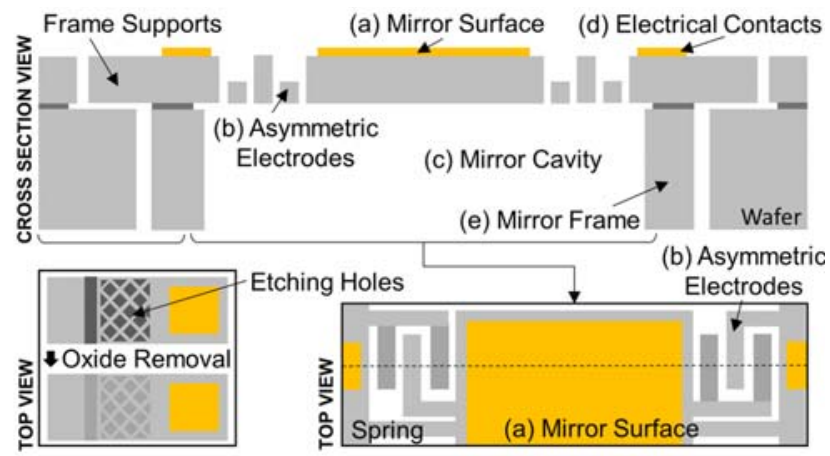

Fig. 5. Cross-section schematic overview of the MEMS mirror components, such as, (a) mirror surface, (b) asymmetric electrodes, (c) mirror cavity, (d) electrical contacts, (e) mirror frame and dicing-free features.

was used to etch the FS silicon layer to different discrete depths in the various regions defined by the multiple GS mask levels. The exposed substrate is etched, in different steps, through DRIE (Deep Reactive Ion Etching), based on the etch time, Fig. 3 (g). Each substrate etching step is followed by a hard-mask thinning process to expose the substrate in the next GS region, Fig. 3 (h), enabling further etching of the substrate, and by a cleaning process of the organic residues due to the DRIE process, Fig. 4, since this during the substrate thinning can become a mask and pattern the silicon.

In the first etching step, a depth of $30 \mu \mathrm{m}$ is defined in the substrate, creating the necessary depth ratio that allows in the next etch process, to achieve the desired substrate topography. The $\mathrm{SiO}_{2}$ mask on top of the thinner electrodes is then removed, exposing the substrate in these regions. Subsequently, during the second etching process, the exposed substrate is etched, resulting in a $20 \mu \mathrm{m}$ thickness in the electrodes, while in the previously partially etched regions the BOX (buried oxide) is reached, decoupling the movable from the fixed asymmetric electrodes, Fig. 3 (i).

The BS was etched (DRIE), to open the mirror cavities to allow a free movement of the mirror, Fig. 3 (j). Finally, the structures were released using hydrogen fluoride (HF) vapor etching, Fig. $3(\mathrm{k})$, removing the sacrificial buried oxide (BOX) layer that is exposed and the remaining oxide hard-masks. Fig. 5 presents a cross-section schematic of the MEMS mirror, illustrating also the HF dicing-free designed features (described in [9]), which consist of FS and BS overlapping trenches around each device and FS grid supports. During the HF process, the BOX layer between the supports

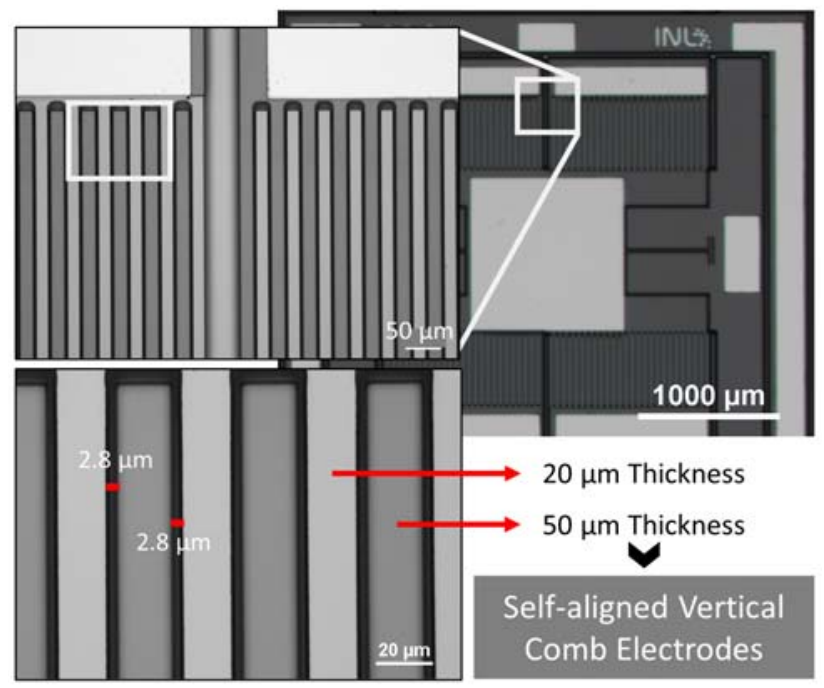

Fig. 6. Optical microscopic images of the fabricated devices, showing self-aligned electrodes with $2.8 \mu \mathrm{m}$ gaps.

and the handle layer is removed, leaving each die suspended by the FS supports on the wafer. These features bypass the need for an extra dicing step, and consequently increasing functional devices yield since these are not submitted to dicing stress.

\section{Fabricated Devices}

The mechanical characterization of the fabricated devices was performed to ensure that all the designed features meet the fabrication specifications.

Using an optical microscope, the fabricated devices were inspected, and as depicted in Fig. 6. The gaps between the two electrodes are entirely aligned, i.e., the gaps are equal in both sides of the electrodes. This is a crucial feature in electrostatic actuation since when an actuation voltage is applied, the in-plane electrostatic forces must be in equilibrium, and only the out-of-plane force should induce the torsional motion of the mirror. It can also be noticed that the gap of $2 \mu \mathrm{m}$ was over etched, resulting in a gap of $2.8 \mu \mathrm{m}$ in the final manufactured devices. This over-etch of the trenches in respect to the mask is typical of DRIE processes and geometry/depth dependent and was accounted for in order to not compromise the performance of the mirror.

All the other mechanical features, such as spring dimensions and mirror area, are within the design specifications with variations below $4 \%$ and $0.08 \%$ respectively.

Fig. 7 presents SEM images of the mirror and its actuators. The two silicon levels are easily identified in both figures (a) and (b), where the lower electrodes present a lighter grey color $(20 \mu \mathrm{m})$, and the full silicon thickness ones a darker grey color $(50 \mu \mathrm{m})$.

The fabricated devices presented the desirable features concluding that this dicing free process combined with this manufacturing process simplified by the grayscale lithography ensured a good yield and the proof of concept of this new low-cost process. 


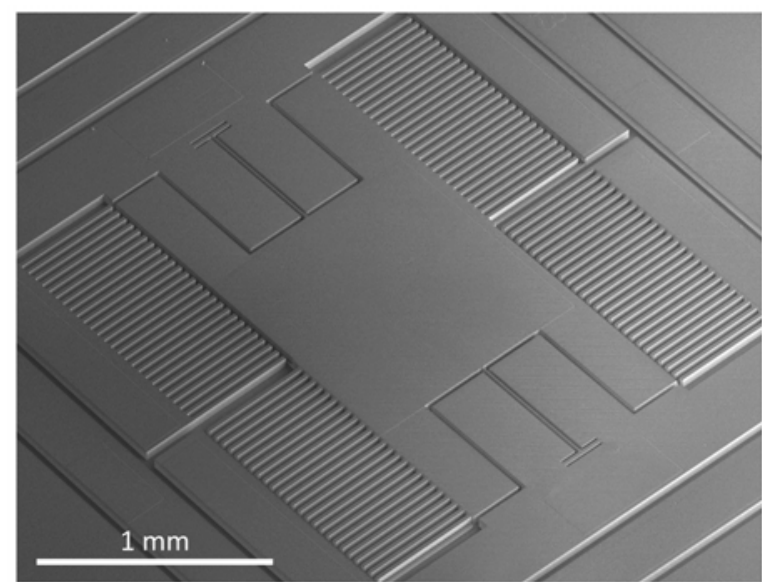

(a)

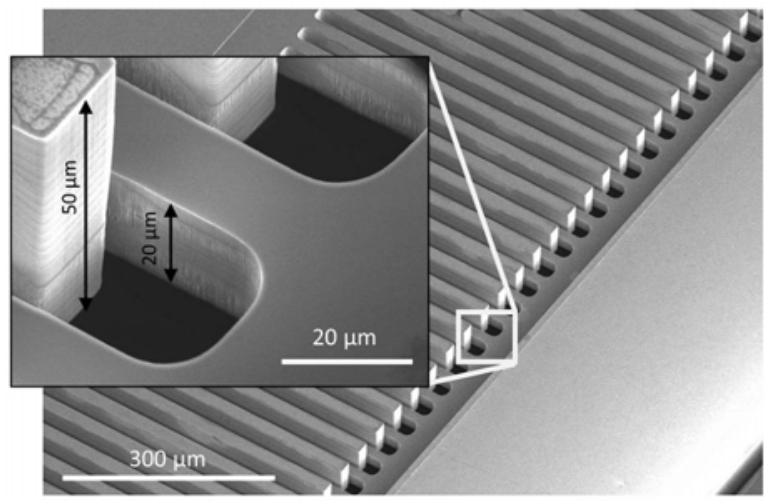

(b)

Fig. 7. SEM images of the fabricated devices: (a) Overall MEMS mirror; (b) Close-view SEM image of the electrostatic actuators.

\section{EXPERIMENTAL CHARACTERIZATION AND RESUlTS}

\section{A. Packaging of MEMS Mirror}

The devices were arranged in a PCB chip-carrier, and the MEMS mirror connections between the chip-carrier pads and the device pads were performed through wire-bonding.

In order to experimentally characterize the device under low pressure, the chip carrier pin-out used was compatible with the vacuum chamber. In these tests, the devices were placed in the vacuum packaging with a glass cover, to enable the laser path to the mirror surface, and using a vacuum pump, the device was submitted to low pressure conditions reducing the damping coefficient and increasing the quality factor.

\section{B. Experimental Setup}

The resonance frequency of the device was experimentally characterized, using a laser scanner vibrometer. Here, it was possible to track the mechanical resonant modes amplitude, when the mirror is electrically excited with low voltage white noise signal. The mirror presented a first resonance mode (torsional) frequency, $f_{0}$, of $838 \mathrm{~Hz}$. This frequency was then used to test and characterize the device with higher voltages to increase the FOV taking advantage of the mechanical amplification due to intrinsic high quality-factor of these type of mechanical structures.

In order to evaluate the mirror performance and FOV, an experimental setup using a laser source (1) and a linear

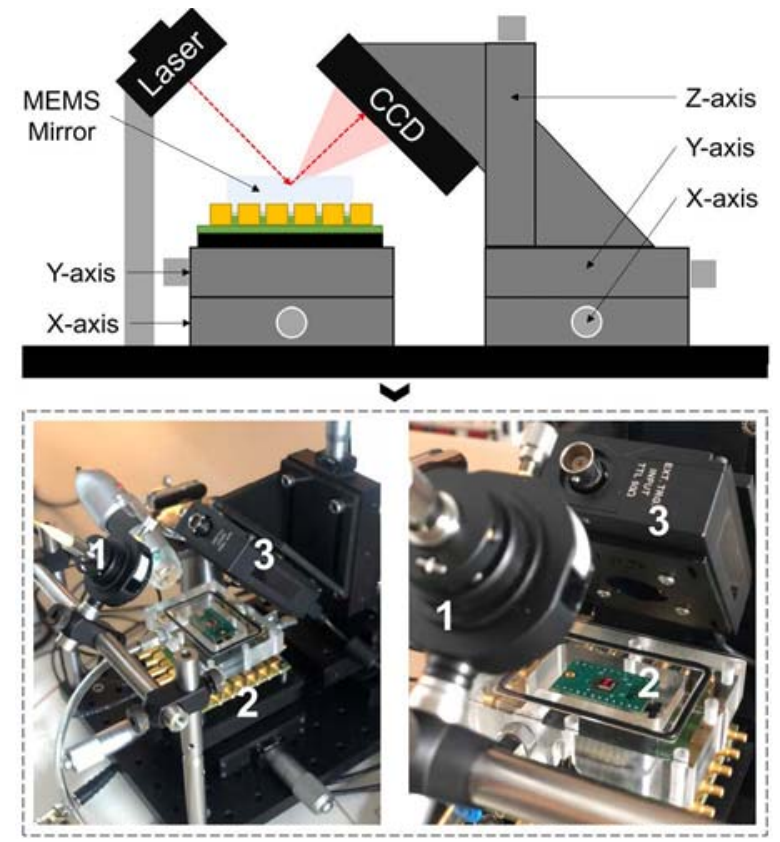

Fig. 8. Experimental setup overview.

CCD camera (3) was assembled to characterize the mirrors for larger deflection angles. An overview schematic of the experimental setup is shown in Fig. 8. This setup has a laser source pointing to the MEMS mirror (2) that can be inside the vacuum packaging to keep the mirror under low pressure. The mirror reflects the beam onto a linear CCD, aligned perpendicular to the beam, enabling a precise measurement of the FOV that the mirror is achieving using simple trigonometric calculations. The mirror deflection angle corresponds exactly to half of the FOV since the incident angle changes with the mirror motion and results in a FOV range twice larger than the mirror deflection angle range. Multiple linear stages are also integrated in the setup, to precisely adjust the distance between the mirror and the linear $\mathrm{CCD}$, aligning the reflected beam with the CCD camera with a minimal error below $13.4 \mathrm{~m}^{\circ}$.

\section{Performance Analysis}

Both static and resonant performance were optically characterized to validate the analytical behavior that was expected to achieve. The performance analysis was divided in two steps:

1) Static Mode: A constant actuation voltage was applied between the asymmetric combs at ambient pressure. In this mode, the air damping coefficient does not affect the mirror behavior. The actuation voltage was varied from $0 \mathrm{~V}$ to $80 \mathrm{~V}$. Fig. 9 presents the correspondent mirror performance, and it is possible to conclude that the mirror deflection angle presents a linear behavior with the applied torque. The quadratic characteristic between the angle and the applied voltage is due to the electrostatic torque since this is proportional to the square of the applied voltage, as stated in equation (3) where $V$ is the voltage applied, $C$ is the capacitance of the combs and $\theta$ is the angle deflection of the mirror.

$$
T_{\text {electrostatic }}=-\frac{1}{2} V^{2} \frac{\partial C}{\partial \theta}
$$




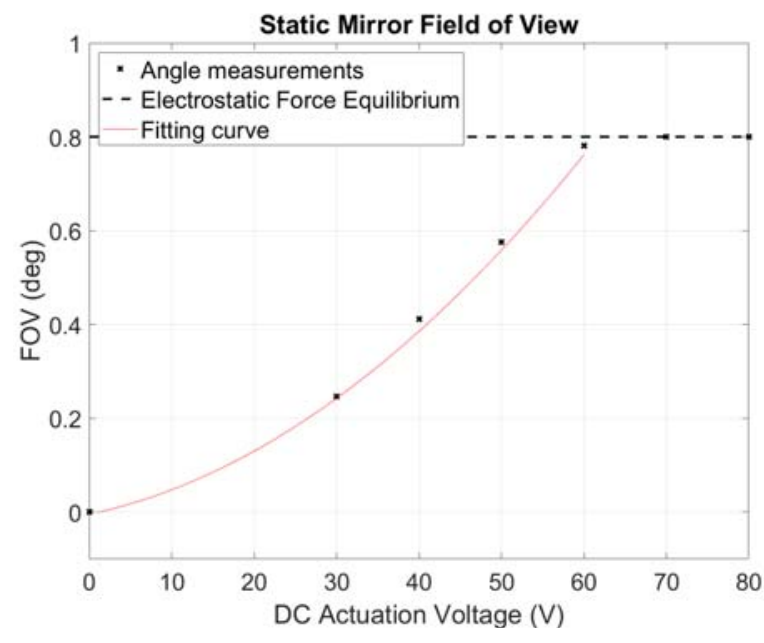

Fig. 9. Static mirror deflection performance, for an applied DC voltage between $0 \mathrm{~V}$ and $80 \mathrm{~V}$, showing a maximum static angle of $0.8^{\circ}$.

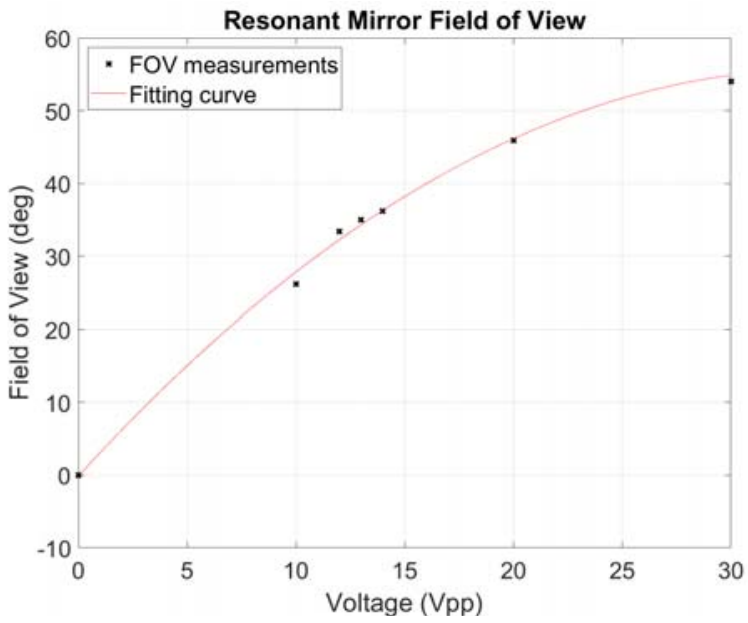

Fig. 10. Resonant mirror FOV, for an applied AC voltage between 0 Vpp and $30 \mathrm{Vpp}$, with a frequency equal to the mirror natural resonance frequency, showing a maximum FOV of $54^{\circ}$, under low pressure.

The mirror achieved, as analytically expected, a static maximum deflection angle of $0.8^{\circ}$ at $70 \mathrm{~V}$ since this is the mechanical alignment between the asymmetric electrodes, and in this point the generated electrostatic forces are in equilibrium.

2) Resonant Mode: In this case, to electrically excite the mirror, a signal generator was used, applying a phase synchronized signal in the mirror right and left actuators independently. This experiment was performed under low pressure (below 5e-5mbar) to reduce the damping coefficient, using the vacuum chamber. The FOV for an applied AC voltage between $0 \mathrm{Vpp}$ and $30 \mathrm{Vpp}$, is presented in Fig. 10. A maximum FOV of $54^{\circ}$ was achieved, with $30 \mathrm{Vpp}$ at the resonance frequency.

\section{CONCLuSion}

The fabrication and characterization of a 1D MEMS mirror for LiDAR application, based on vertical asymmetric electrostatic actuation, was presented. It was introduced a novel micromachining technique to create the asymmetry between the vertical comb actuators, merging typical SOI-based bulk micromachining processes and grayscale lithography. This technique simplifies and reduces the overall fabrication costs by also reducing the number of required lithography steps. Moreover, it bypasses the misalignment problems arising from using separate lithography steps for the different sets of electrodes with different heights.

The asymmetric combs electrodes have consistent and reproducible $2.8 \mu \mathrm{m}$ gap, which proves this process to be an effective way to fabricate self-aligned and robust structures with different silicon levels, enabling both operation modes of the fabricated devices, static mode and resonant mode.

The mirrors were optically characterized and resonantly actuated, in a low-pressure chamber to reduce the damping coefficient, achieving a reflection FOV of $54^{\circ}$, at a resonance frequency of $838 \mathrm{~Hz}$. When operated in the static mode, these devices achieved a maximum FOV of $0.8^{\circ}$ at $70 \mathrm{~V}$, corresponding also to the combs' electrostatic force alignment angle due to the different electrodes' heights.

In the future, this novel self-aligned multilevel micromachining process will be used in a 2D MEMS mirror that can be optimized in order to fabricate devices with more than three levels, as well as to enable the patterning of different materials, such as the metal layer and the silicon structures in a single GS mask. A typical fabrication process for this specific device would require four different lithographic processes. This has the potential to reduce the number lithography steps required for processing such MEMS structures to two (one for the frontside and another for the backside of the SOI wafer). This process could also be optimized to create more levels in the SOI device layer, such as to create membranes or springs with thinner or varying heights, which are self-aligned with the rest of the MEMS structure.

\section{REFERENCES}

[1] S. T. S. Holmstrom, U. Baran, and H. Urey, "MEMS laser scanners: A review," J. Microelectromech. Syst., vol. 23, no. 2, pp. 259-275, Apr. 2014.

[2] M. J. Madou, Fundamentals of Microfabrication: The Science of Miniaturization, 2nd ed. Boca Raton, FL, USA: CRC Press, 2002.

[3] J. Cheng et al., "An auto-aligned vertical comb drive for low-cost variable optical attenuators," in Proc. 19th Int. Conf. Solid-State Sens., Actuators Microsystems (TRANSDUCERS), Kaohsiung, Taiwan, Jun. 2017, pp. 622-625.

[4] Z. Hailu, S. He, and R. B. Mrad, "A novel vertical comb-drive electrostatic actuator using a one layer process," J. Micromecha. Microeng., vol. 24, no. 11, pp. 1-11, 2014.

[5] H. Hamaguchi, K. Sugano, T. Tsuchiya, and O. Tabata, "A differential capacitive three-axis SOI accelerometer using vertical comb electrodes," in Proc. Int. Solid-State Sens., Actuators Microsyst. Conf. (TRANSDUCERS), Lyon, France, 2007, pp. 1483-1486.

[6] T. Tsuchiya and H. Funabashi, "A z-axis differential capacitive SOI accelerometer with vertical comb electrodes," Sens. Actuators A, Phys., vol. 116, no. 3, pp. 378-383, Oct. 2004.

[7] H.-T. Su, G.-L. Luo, J. Hsieh, Y.-C. Fu, and W. Fang, "Process platform for high aspect-ratio self-aligned vertical comb actuators," in Proc. 18th Int. Conf. Solid-State Sens., Actuators Microsyst. (TRANSDUCERS), Anchorage, Alaska, Jun. 2015, pp. 1342-1345.

[8] Y. Fan, C. Cui, H. Yu, W. Shen, Z. Wang, and J. Li, "An electrostatic vertical comb-drive micromirror with self-aligned assembly," in Proc. IEEE 12th Int. Conf. Nano/Micro Engineered Mol. Syst. (NEMS), Los Angeles, CA, USA, Apr. 2017, pp. 268-272. 
[9] I. S. Garcia, E. E. Moreira, R. A. Dias, J. Gaspar, F. S. Alves, and L. A. Rocha, "Sub-micron mems accelerometer with handle-layer patterning for damping enhancement using time transduction," in Proc. 20th Int. Conf. Solid-State Sens., Actuators Microsyst. Eurosensors XXXIII (TRANSDUCERS EUROSENSORS XXXIII), Berlin, Germany, Jun. 2019, pp. 2045-2048.

[10] Q. Deng, Y. Yang, H. Gao, Y. Zhou, Y. He, and S. Hu, "Fabrication of micro-optics elements with arbitrary surface profiles based on one-step maskless grayscale lithography," Micromachines, vol. 8, no. 10, p. 314 Oct. 2017.

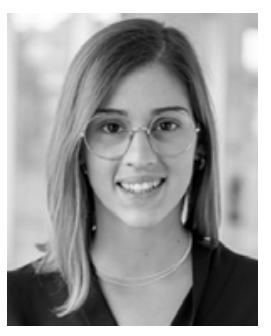

Inês S. Garcia received the M.Sc. degree in industrial electronics and computers engineering from the University of Minho, Guimarães, Portugal, in 2019, where she is currently pursuing the Ph.D. degree in electronics and computers engineering.

She is currently a Micro-Optical-ElectroMechanical Systems (MOEMS) Research Engineer with the Micro and Nanofabrication Department, INL. She has a strong background in automation, control and robotics, and microtechnologies. She joined, in 2018, the Microfabrication and Exploratory Nanotechnology Group, International Iberian Nanotechnology Laboratory (INL), where she developed her master's thesis on High Precision MEMS Accelerometers for Microsatellites. Her research is focused on the design, fabrication, and characterization of MOEMS and MEMS devices.

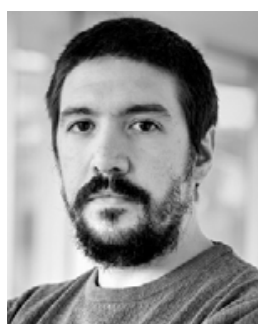

Carlos Ferreira (Member, IEEE) received the master's degree in electronics engineering from the University of Minho, Portugal, in 2016, where he is currently pursuing the Ph.D. degree in advanced engineering systems for industry.

His research is centered on inertial and optical MEMS design and characterization combined with electronic design for closed-loop control and high-speed test, using FPGA and ARM-based embedded systems.

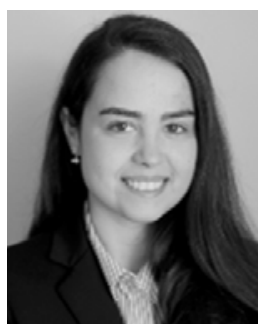

Joana D. Santos received the B.Sc. degree in electronics engineering and the M.Sc. degree in bioengineering and nanosystems from the Instituto Superior Técnico (IST).

She is currently a Cleanroom Research Engineer with the Microfabrication and Exploratory Nanotechnology Group, International Iberian Nanotechnology Laboratory (INL). She has worked in micro and nanotechnology research at the Instituto de Engenharia de Sistemas e Computadores Microsistemas e Nanotecnologias (INESC-MN), Lisbon, where she completed her master's thesis on Methodologies for Monolithic Integration and 3D TSV Microfabrication for Chip Integration With CMOS She has an extensive experience in cleanroom processes being responsible at INL for operation, maintenance, and training of multiple equipment and processes, including optical lithography, wet etch, and metrology tools.

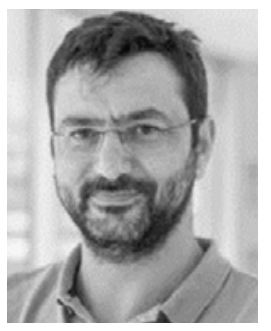

Marco Martins received the degree in electrical and computer engineering from the Instituto Superior Técnico (IST), Lisbon. He has worked in semiconductor industry (Chipidea and Silicon Labs) as a Test Engineer/Application Engineer for 12 years and currently belongs to INL's staff of engineers performing application engineer functions since 2012 . He works actively in the design and development of electronic and software systems from concept to production with special emphasis on the design of very low-noise analog systems for signal conditioning in the hardware area, software development for signal processing, and the integration of embedded systems.

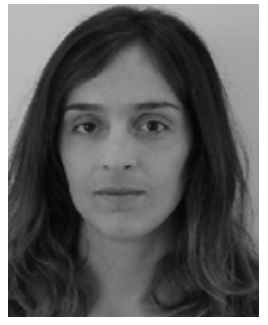

Rosana A. Dias received the Ph.D. degree in MEMS, focusing on pull-in time accelerometers from the University of Minho and The University of the British Columbia.

She is currently a Staff Researcher with the Microfabrication and Exploratory Nanotechnology Group, INL. Her expertise concerns the development of sensors and actuators to meet the technical challenges faced by society and industry. She has large experience in multidomain modeling, design, microfabrication processing, and lab testing of MEMS devices. Her research interests range from mechanical sensors (e.g., acceleration and magnetic field sensing) to energy harvesting devices (piezoelectric AlN and EMF-based induction), flexible substrate-based applications and optical devices (micromirrors, filters, and optical nanostructures). She has developed a gas viscosity sensing application at and in collaboration with the Delft University of Technology. She has also been the main responsible, as a Post-Doctoral Researcher, for the design of a MEMS magnetometer for space applications, within the European Space Agency Project.

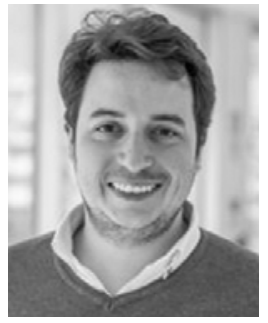

Diogo E. Aguiam (Member, IEEE) received the M.Sc. degree in electronics engineering from the Instituto Superior Técnico and the double Ph.D. degrees in engineering physics from Ghent University and the University of Lisbon.

$\mathrm{He}$ is currently a Staff Researcher with the Microfabrication and Exploratory Nanotechnology Group, INL, contributing to the development of electronic instrumentation, embedded systems, and data processing techniques. He worked on a standalone embedded system for eddy currents nondestructive testing of faults in metallic parts at the Instituto Superior Técnico. He has developed microwave reflectometry diagnostics for nuclear fusion plasma electron density profile measurements at the University of Lisbon. During his Ph.D., he worked between the Instituto de Plasmas e Fusão Nuclear, Lisbon, Portugal, and the Max Planck Institute for Plasma Physics, Garching, Germany, where he collaborated in large international teams and responsible for diagnostic maintenance and development of data analysis software.

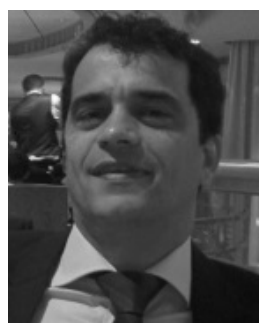

Jorge Cabral (Member, IEEE) received the Ph.D. degree in microsystems technology from Imperial College London, London, U.K. He is currently an Assistant Professor with the University of Minho, Portugal. His research interests include embedded systems applications and he is in charge of several research projects in this field.

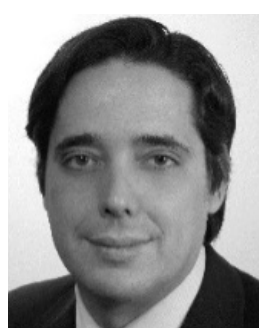

João Gaspar (Member, IEEE) received the Ph.D. degree in materials engineering from the INESC Microsystems and Nanotechnologies, Instituto Superior Técnico (IST), Lisbon, in 2004.

He focused on thin-film silicon MEMS at IST He joined the Microsystems Materials Laboratory (MML), Department of Microsystems Engineering (IMTEK), University of Freiburg, Germany, in 2004, as a Post-Doctoral Fellow for two years. In 2006, he was promoted to a Lecturer and the Group Leader with MML, IMTEK, where he held a position until joining the International Iberian Nanotechnology Laboratory (INL), Braga, in 2011. He is currently the Group Leader of the Microfabrication and Exploratory Nanotechnology Group, INL. His research interests include the microfabrication processes and advanced silicon micromachining, MPW runs, high-throughput wafer-scale testing and reliability of MEMS, resonance and optical applications, microenergy harvesting, and microneedles/capillary arrays for intracellular recording plus post-CMOS processing. 